\title{
Dose-Response Relationship in Differentiated Thyroid Cancer Patients Undergoing Radioiodine Treatment Assessed by Means of ${ }^{124}$ I PET/CT
}

\author{
Roel Wierts ${ }^{1}$, Boudewijn Brans ${ }^{1}$, Bas Havekes ${ }^{1}$, Gerrit J. Kemerink ${ }^{1}$, Servais G. Halders ${ }^{1}$, Nicolaas N. Schaper ${ }^{1}$, \\ Walter H. Backes ${ }^{1}$, Felix M. Mottaghy ${ }^{1,2}$, and Walter Jentzen ${ }^{3}$ \\ ${ }^{1}$ Maastricht University Medical Center, Maastricht, The Netherlands; ${ }^{2}$ University Hospital RWTH Aachen University, Aachen, \\ Germany; and ${ }^{3}$ Klinik für Nuklearmedizin, Universität Duisburg-Essen, Essen, Germany
}

\begin{abstract}
The dose-response relationship in a fixed-activity approach generally applied in the treatment of differentiated thyroid cancer was assessed using ${ }^{124}$ I PET/CT. Methods: Pretherapeutic ${ }^{124}$ I PET/ $\mathrm{CT}$ images of 47 patients scheduled for radioiodine therapy were retrospectively analyzed. ${ }^{124}$ I PET/CT images were acquired 24 and $96 \mathrm{~h}$ after oral administration of approximately $28 \mathrm{MBq}$ of ${ }^{124}$ I-sodium iodide. Lesions were identified as thyroid remnants or metastases (lymph node, lung, bone). After a neoteric segmentation technique allowing accurate volume estimation down to the 124I PET spatial resolution of $0.15 \mathrm{~mL}$ was applied, lesions were divided into a known-volume group and a small-volume group. For the known-volume group, average lesion-absorbed dose (AD) values were calculated, whereas for the small-volume group a minimum lesion $A D$ was estimated. Lesion response was determined on the basis of ${ }^{124}$ I PET/CT and ${ }^{131}$ I SPECT/CT follow-up images. A lesion not detectable on any of the follow-up images was considered a completely responding lesion. Differences in lesion $A D$ estimations between completely and incompletely responding lesions were evaluated by Mann-Whitney $U$ test. Moreover, receiver-operating-characteristic curves were used to test the performance of pretherapeutic ${ }^{124}$ I PET/CT lesion AD for prediction of complete lesion response. Results: In the approach of fixed radioiodine activity $(3.0 \pm 1.0 \mathrm{GBq}), 89 \%$ of thyroid remnants and $69 \%$ of metastases responded completely. Except for the small-volume groups, the lesion AD of completely responding lesions was significantly higher than that of incompletely responding lesions. Using receiver-operating-characteristic curve analysis, it was shown that for the known-volume group, pretherapeutic ${ }^{124}$ I PET/CT lesion dosimetry can be used as a prognostic tool to predict lesion-based ${ }^{131}$ I therapy response with an area under the curve of 0.76 for remnants and 0.97 for metastases. The corresponding lesion $A D$ threshold value maximizing correct complete response prediction was 90 Gy for remnants and 40 Gy for metastases. Conclusion: In a fixed-activity approach, a statistically significant dose-response relationship for both thyroid remnants and metastases using pretherapeutic ${ }^{124}$ I PET/CT lesion dosimetry was found. The findings may be useful in patient management.
\end{abstract}

Received Oct. 23, 2015; revision accepted Feb. 5, 2016.

For correspondence or reprints contact: Roel Wierts, Maastricht University

Medical Centre, P. Debyelaan 25, 6229 HX Maastricht, The Netherlands.

E-mail: roel.wierts@mumc.nl

Published online Feb. 25, 2016.

COPYRIGHT (c) 2016 by the Society of Nuclear Medicine and Molecular Imaging, Inc.
Key Words: radioiodine therapy; thyroid carcinoma; lesion-response relationship; ${ }^{124}$; dosimetry

J Nucl Med 2016; 57:1027-1032

DOI: $10.2967 /$ jnumed.115.168799

$\mathbf{R}$ adioiodine therapy is the standard adjuvant therapy after total thyroidectomy in patients with differentiated thyroid cancer (DTC) $(1,2)$. The purpose of radioiodine therapy is 2-fold: ablation of thyroid remnants and treatment of radioiodine-avid metastases.

The generally accepted threshold for lesion-absorbed dose (AD) to achieve high therapy response is 85 Gy for metastases and $300 \mathrm{~Gy}$ for thyroid remnants $(3,4)$. To predict therapy response, a reliable $\mathrm{AD}$ estimation requires, inter alia, accurate lesion volume determination, which is hampered by the often small lesion volumes with respect to the spatial resolution of ${ }^{131}$ I scintigraphy and lesion segmentation difficulties encountered in ultrasonography, CT, or other imaging modalities $(1,5)$. As a result, personalized radioiodine therapy based on lesion dosimetry is not globally adopted. In general, a fixed ${ }^{131} \mathrm{I}$ activity is administered on the basis of disease characteristics and patient age, with the risk of under- or overtreatment (6).

Several groups have concluded that quantitation of ${ }^{124}$ I PET images is feasible (7-11). Compared with ${ }^{131} \mathrm{I}$ SPECT, ${ }^{124} \mathrm{I}$ PET imaging offers a higher image spatial resolution, increased counting rate sensitivity, and higher quantitative capacity, resulting in the application of pretherapeutic ${ }^{124} \mathrm{I}$ PET/CT-based lesion dosimetry (12-17).

To date, the only study assessing the dose-response relationship using ${ }^{124} \mathrm{I}$ PET/CT in a larger number of patients was recently published by Jentzen et al. (17). Pretherapeutic ${ }^{124} \mathrm{I}$ PET/CT lesion $\mathrm{AD}$ estimation and toxicity assessment were taken into consideration in patient management, allowing the administration of an optimized therapeutic activity $(8.0 \pm 4.3 \mathrm{GBq})$. However, using a fixed-activity approach in the absence of toxicity assessment generally results in lower administered ${ }^{131}$ I activity than the optimized therapeutic activity. Consequently, it is expected that the lesion ADs in the fixed-activity approach are lower than the values published by Jentzen et al. (17), possibly resulting in more lesions that receive a lesion $\mathrm{AD}$ below the established threshold values.

Therefore, the aim of this study was to assess the dose-response relationship in an approach of a fixed therapeutic ${ }^{131} \mathrm{I}$ activity in a large group of patients using pretherapeutic ${ }^{124}$ I PET/CT lesion dosimetry. Moreover, a new segmentation technique allowing volume 
estimation down to the PET spatial resolution of ${ }^{124}$ I was used (18). In addition, we assessed the feasibility of performing pretherapeutic ${ }^{124}$ I PET/CT lesion dosimetry as a prognostic tool to predict therapy response in DTC patients in a fixed-activity approach.

\section{MATERIALS AND METHODS}

\section{Patient Population}

This study was approved by the institutional review board, and the requirement to obtain informed consent was waived. In the Maastricht University Medical Centre, pretherapeutic imaging with ${ }^{124} \mathrm{I}$ was introduced as a clinical standard in 2007. A retrospective analysis of DTC patients who underwent ${ }^{124}$ I PET/CT examinations, followed by ${ }^{131}$ I therapy, between January 2007 and December 2012 was performed. All patients had histologically confirmed papillary or follicular DTC and underwent total thyroidectomy before ${ }^{124}$ I PET/CT examinations. None of the patients received additional treatment such as surgery or external-beam radiation therapy. Patients were included if (posttherapy) follow-up imaging with either (pretherapeutic) ${ }^{124} \mathrm{I}$ PET/CT or ${ }^{131}$ I planar whole-body scintigraphy and ${ }^{131}$ I SPECT/CT as part the radioiodine therapy was available. For patients who underwent multiple radioiodine therapies preceded by ${ }^{124} \mathrm{I}$ PET/CT examinations, only the data of the first radioiodine therapy were included. Patient TNM status was characterized in 4 stages according to the American Joint Committee on Cancer Staging Atlas (19). Moreover, the maximum thyroglobulin value recorded within 1 y after ${ }^{131} \mathrm{I}$ ablation therapy was reported. Until March 2011, thyroglobulin was measured using a time-resolved fluoroimmunoassay (Autodelfia; PerkinElmer) with a limit of detection of $0.5 \mathrm{pmol} / \mathrm{L}$. From March 2011, thyroglobulin was measured using a time-resolved amplified cryptate emission assay (Brahms $\mathrm{GmbH}$; Thermo Fisher) with a limit of detection of $0.25 \mathrm{pmol} / \mathrm{L}$. Abnormal (positive) results were recorded if thyroglobulin was greater than $1.5 \mathrm{pmol} / \mathrm{L}$. In the case of incomplete recovery of the antibody used in the assay, additional testing was done for specific antithyroglobulin antibodies.

Patient preparation was done by thyroid hormone withdrawal or recombinant human thyroid-stimulating hormone (rhTSH) and was similar for the pre- and follow-up ${ }^{124}$ I PET/CT examinations. In the case of thyroid hormone withdrawal, patients were withdrawn from thyroxine medication for 4-6 wk or remained without medication postoperatively. In the cases of rhTSH preparation, $0.9 \mathrm{mg}$ of thyrotropin alfa or rhTSH (Thyrogen; Genzyme Ltd.) was injected intramuscularly on days 1 and 2, afterward ${ }^{124} \mathrm{I}$ was orally administered on day 4, and ${ }^{124} \mathrm{I}$ PET/CT was performed on day 5 (24 h after ${ }^{124} \mathrm{I}$ administration) and day 8 ( $96 \mathrm{~h}$ after ${ }^{124} \mathrm{I}$ administration). For radioiodine therapy, a fixed-activity protocol was performed according to the Dutch guidelines, using $2.8 \mathrm{GBq}(75 \mathrm{mCi})$ for simple thyroid remnant ablation and $5.6 \mathrm{GBq}(150 \mathrm{mCi})$ for regional nodal disease or distant metastases.

\section{Image Acquisition and Reconstruction}

${ }^{124}$ I PET/CT was performed in 3-dimensional mode using a PET camera equipped with time-of-flight (Gemini TF PET/64-slice CT scanner; Philips) at 24 and $96 \mathrm{~h}$ after oral administration of $28.0 \pm 3.3 \mathrm{MBq}$ of ${ }^{124} \mathrm{I}$-sodium iodide. ${ }^{124} \mathrm{I}$ radioactivity was measured using a $3-\mathrm{mL}$ syringe filled with approximately $1 \mathrm{~mL}$ of ${ }^{124} \mathrm{I}$ solution that was placed in a validated dose calibrator (Isomed 2000; Nuklear Medizin Technik $\mathrm{GmbH}) .{ }^{124} \mathrm{I}$ PET scans were acquired from the head, neck, and thorax comprising 4-5 bed positions of 4 min each. In selected cases, imaging was continued until the pelvis. A low-dose spiral CT scan from the head to thigh was acquired (tube voltage, $120 \mathrm{kVp}$; effective tube current, $30 \mathrm{mAs}$; slice thickness, $4 \mathrm{~mm}$ ), followed by the PET acquisition and supplemental high-dose CT without contrast (neck, $120 \mathrm{keV}$; $150 \mathrm{mAs}$; slice thickness, $2 \mathrm{~mm}$; increment, 1.8; and thorax, $120 \mathrm{keV}$; $175 \mathrm{mAs}$; slice thickness, $5 \mathrm{~mm}$; increment, 4.0). All CT images were reconstructed using the filtered backprojection algorithm. PET images were reconstructed using the line of response-based, time-of-flight reconstruction algorithm provided by the manufacturer with a voxel size of $4 \times 4 \times$ $4 \mathrm{~mm}^{3}$. In addition, for accurate lesion volume assessment and quantification purposes, PET images were retrospectively reconstructed with a voxel size of $2 \times 2 \times 2 \mathrm{~mm}^{3}$. For all PET images, standard corrections for attenuation, scatter, decay, and dead-time were performed.

The ${ }^{131}$ I whole-body scintigraphy was made as a total-body scan from top until toe using a table speed of $10 \mathrm{~cm} / \mathrm{min}$. SPECT/CT was acquired immediately after whole-body scintigraphy. ${ }^{131} \mathrm{I}$ SPECT/CT was acquired using a standard SPECT/CT camera (Precedence SPECT/6-slice CT scanner; Philips) equipped with dual 1.6-cm $\gamma$ detectors with high-energy general-purpose collimators. SPECT data were obtained by a noncircular orbit, a $128 \times 128$ matrix (voxel size, $4.7 \times 4.7 \times 4.7 \mathrm{~mm}^{3}$ ), and 32 angles over $180^{\circ}$ and 45 s per stop, using a $364-\mathrm{keV}$ photo peak with $10 \%$ window (total acquisition time, $24 \mathrm{~min}$ ). Reconstruction space and width was $3 \mathrm{~mm}$ using the Philips Astonish algorithm.

\section{Lesion Volume Calculation}

The volume of each lesion with focal uptake, present on both of the pretherapeutic ${ }^{124}$ I PET images (24 and $96 \mathrm{~h}$ after administration), was semiautomatically determined using an in-house-built software algorithm (Matlab; The MathWorks) based on a recently published iterative thresholding method (18). The method assumes a spherically shaped lesion with homogeneous ${ }^{124} \mathrm{I}$ uptake. Accounting for the reconstructed ${ }^{124}$ I PET spatial resolution of $6.7 \mathrm{~mm}$ (expressed as full width at half maximum), background-corrected relative boundary-reproducing values used for lesion delineation were calculated. The smallest diameter or volume that can be determined using this method corresponds to the PET spatial resolution or its equivalent sphere volume of $0.15 \mathrm{~mL}$. Consequently, lesions were classified into 2 groups in line with a previous study (17): lesions with reliable volume estimation larger than $0.15 \mathrm{~mL}$, the so-called known-volume group, and lesions with a volume smaller than $0.15 \mathrm{~mL}$, the small-volume group. For the known-volume group, the lesion volume was calculated as the average value of the lesion volume determined on the 24- and 96-h ${ }^{124}$ I PET images. Lesions for which the average lesion volume differed more than $30 \%$ with respect to the 24- or 96-h volume were excluded. Lesions for which either the $24-$ or the $96-\mathrm{h}$ volume was smaller than $0.15 \mathrm{~mL}$ were classified into the small-volume group. For the small-volume group, the lesion volume used for lesion $\mathrm{AD}$ estimation was assumed to be equal to the PET spatial resolution volume of $0.15 \mathrm{~mL}$.

\section{Lesion-Absorbed Dose Estimation}

For each ${ }^{124}$ I PET image, lesion uptake was calculated as the average activity concentration of the segmented volume, corrected for partialvolume effect, using measured (absolute) recovery coefficients (18), which effectively corrects for prompt $\gamma$-coincidence effect as well (20). Assuming identical ${ }^{124} \mathrm{I}$ and ${ }^{131} \mathrm{I}$ pharmacokinetics, half-life correction was performed on the ${ }^{124}$ I activity concentration to assess the projected ${ }^{131}$ I activity concentration. ${ }^{131} \mathrm{I}$ residence time was determined according to the adapted 2-points approach (21). In the case the effective half-life was less than the physical ${ }^{131}$ I half-life, the lesion time-activity curve was parameterized using a combination of a linear uptake function and a monoexponential decay function (interception time at $8 \mathrm{~h}$ after ${ }^{131}$ I administration). In the case the effective half-life was greater than the ${ }^{131}$ I physical half-life, physical decay was assumed beyond the 96$\mathrm{h}$ time point. The (self-irradiation) lesion $\mathrm{AD}$ was calculated using the sphere model in the Olinda software package (Olinda version 1.1; Vanderbilt University) (22). For all lesions, a density of $1.0 \mathrm{~g} / \mathrm{mL}$ was used, resulting in an average and a minimum lesion $\mathrm{AD}$ per administered ${ }^{131}$ I activity for the known-volume and small-volume 
groups, respectively. The predicted average and minimum lesion AD delivered in radioiodine therapy were estimated by multiplication with the therapeutic ${ }^{131}$ I activity.

\section{Therapy Response Assessment}

Lesion-Based Analysis. Lesions were classified as either thyroid remnants or metastases including lymph node or distant metastases (lymph node, lung, bone). Both the thyroid remnants and the metastases were subdivided into a known-volume group and a small-volume group. Each individual lesion was defined either as completely or as incompletely responding by an experienced nuclear medicine physician and medical physicist. Specifically, the completely responding lesion did not show ${ }^{124} \mathrm{I}$ uptake on subsequent follow-up scanning within $1 \mathrm{y}$ and no ${ }^{131} \mathrm{I}$ uptake on subsequent posttherapeutic planar and SPECT/CT scanning. In contrast, the incompletely responding lesion did show focal ${ }^{124} \mathrm{I}$ or ${ }^{131} \mathrm{I}$ uptake on the day-4 scan (in the absence of significant level of background noise), not contributable to physiologic uptake according to visual assessment. For each lesion group, the predicted lesion AD in radioiodine therapy of the completely responding lesions was compared with that of the incompletely responding lesions. Moreover, the performance of pretherapeutic ${ }^{124} \mathrm{I}$ PET/CT to predict complete lesion response was assessed using receiver-operating-characteristic (ROC) curve analysis (23).

Patient-Based Analysis. Patients were classified as incompletely responding if persisting disease after radioiodine therapy was demonstrated, either by functional imaging $\left({ }^{124} \mathrm{I}\right.$ PET/CT, ${ }^{131} \mathrm{I}$ SPECT/CT, ${ }^{18} \mathrm{~F}-\mathrm{FDG}$ ), anatomic imaging (ultrasonography, MRI), histology, or increased thyroglobulin value. Otherwise patients were classified as completely responding. The average patient-based lesion $\mathrm{AD}$ was calculated as the mean lesion AD of all radioiodine-avid lesions observed per patient.

\section{Statistics}

Statistical analysis was performed using SPSS version 22 (IBM Corp.). Descriptive data are shown as mean \pm SD (median; range). Differences between 2 groups were assessed using the MannWhitney $U$ test. Values for $P$ less than 0.05 were considered statistically significant. ROC curves were used to test the performance of pretherapeutic ${ }^{124} \mathrm{I}$ PET/CT lesion AD for the prediction of complete lesion response.

\section{RESULTS}

\section{Patient and Lesion Characteristics}

Detailed patient characteristics are provided in Table 1. Of the in total 67 patients satisfying the inclusion criteria, 1 patient was excluded because this patient received a diagnostic CT with iodinecontaining contrast agent before the ${ }^{124} \mathrm{I}$ PET/CT scan. Three patients were excluded because of the lack of either the 24- or the 96-h ${ }^{124}$ I PET/CT examination. Furthermore, 12 patients were excluded because of the unavailability of the PET raw data required for the additional retrospective $2 \times 2 \times 2 \mathrm{~mm}^{3}$ voxel image reconstruction. Four patients did not show any visible lesions on the ${ }^{124}$ I PET images. Of the remaining 47 patients, 29 patients were classified as completely responding and 18 patients as incompletely responding.

TABLE 1

Characteristics of Analyzed $(n=47)$ Patients

\begin{tabular}{|c|c|c|}
\hline Characteristic & Completely responding & Incompletely responding \\
\hline Age (y) & $51 \pm 11(52 ; 23-70)$ & $50 \pm 18(53 ; 18-79)$ \\
\hline \multicolumn{3}{|l|}{ Sex } \\
\hline Male & 4 & 7 \\
\hline Female & 25 & 11 \\
\hline \multicolumn{3}{|l|}{ Number of lesions } \\
\hline Thyroid remnants & 74 & 49 \\
\hline Metastases & 6 & 39 \\
\hline \multicolumn{3}{|l|}{ Variant } \\
\hline Papillary & 21 & 11 \\
\hline Follicular & 21 & 11 \\
\hline \multicolumn{3}{|l|}{ TNM stage } \\
\hline 1 & 13 & 8 \\
\hline 2 & 4 & - \\
\hline 3 & 12 & 4 \\
\hline 4 & - & 6 \\
\hline Maximum thyroglobulin value in $\mathrm{FU}(\mathrm{pmol} / \mathrm{L})$ & $11 \pm 55(<0.5 ; 0.25-290)$ & $2.5 \cdot 10^{3} \pm 7.0 \cdot 10^{3}\left(5.7 ;<0.2-2.5 \cdot 10^{4}\right)$ \\
\hline \multicolumn{3}{|l|}{ First ${ }^{131}$ | radionuclide therapy } \\
\hline Yes & 26 & 16 \\
\hline No & 3 & 2 \\
\hline Single therapeutic ${ }^{131} \mid$ activity $(\mathrm{GBq})$ & $3.0 \pm 0.7(2.8 ; 2.8-5.5)$ & $3.0 \pm 1.0(2.8 ; 1.1-5.5)$ \\
\hline Follow-up period (mo) & $19 \pm 16(10 ; 4.5-61)$ & $17 \pm 17(9 ; 3.5-64)$ \\
\hline
\end{tabular}

Descriptive data are shown as mean $\pm S D$, with median and range in parentheses. 
TABLE 2

Overview of Analyzed Lesions

\begin{tabular}{|ccc}
\hline Characteristic & $\begin{array}{c}\text { Completely } \\
\text { responding }\end{array}$ & $\begin{array}{c}\text { Incompletely } \\
\text { responding }\end{array}$ \\
\hline Thyroid remnants & 109 & 14 \\
\hline Known-volume group & 39 & 8 \\
\hline Paratracheal & 12 & 5 \\
\hline Thyroid bed & 7 & - \\
\hline Cervical high & 20 & 3 \\
\hline Small-volume group & 70 & 6 \\
\hline Paratracheal & 45 & 1 \\
\hline Thyroid bed & 11 & 1 \\
\hline Cervical high & 14 & 14 \\
\hline Metastases & 31 & 12 \\
\hline Known-volume group & 6 & - \\
\hline Thyroid bed & - & 1 \\
\hline Neck lateral (II-V) & 5 & - \\
\hline Cervical high & - & - \\
\hline Cervical low (VI) & 1 & 11 \\
\hline Distant & - & 2 \\
\hline Small-volume group & 25 & 1 \\
\hline Thyroid bed & 2 & - \\
\hline Neck lateral (II-V) & 18 & - \\
\hline Cervical high & 4 & - \\
\hline Cervical low & 1 & - \\
\hline Distant & - & 1 \\
\hline & & - \\
\hline
\end{tabular}

Detailed lesion characteristics are given in Table 2. In total, 168 lesion were suitable for AD estimation. Nine lesions were excluded because of lesion volume discrepancies between the 24and 96-h scans. For the thyroid remnants and metastases, $89 \%$
$(109 / 123)$ and $69 \%(31 / 45)$ of the lesions showed a complete response, respectively.

\section{Lesion-Based Therapy Response}

Figure 1 illustrates the calculated lesion $\mathrm{AD}$ in radioiodine therapy for both thyroid remnants and metastases for all lesions (including known- and small-volume group), the known-volume group, and the small-volume group. A statistically significant higher lesion $\mathrm{AD}$ was observed for the completely responding lesions than the incompletely responding lesions for all lesions and the known-volume group, but not for the small-volume remnants group. Because there were only 2 incompletely responding lesions in the small-volume metastases group, no statistical tests were performed for this group. Detailed lesion data are provided in the supplemental materials (available at http://jnm. snmjournals.org).

Figure 2 shows the ROC curves for both the thyroid remnants and the metastases. Except for the metastatic small-volume group, all area under the curve values were significantly higher than 0.5 . The area under the curve values were highest for the known-volume group. The arrow in Figure 2 corresponds to the established threshold values for the lesion $\mathrm{AD}$ of $300 \mathrm{~Gy}$ for thyroid remnants and 85 Gy for metastases $(3,4)$. For the known-volume group, these threshold values resulted in sensitivity values of 0.69 and 0.67 and specificity values of 0.75 and 0.92 for the thyroid remnants and metastases, respectively. The Youden index (24), defined as the value that gives the maximum correct classification, was calculated as being 90 Gy for thyroid remnants and 40 Gy for metastases, resulting in respective sensitivity values of 0.85 and 1.0 and specificity values of 0.75 and 0.92 .

\section{Patient-Based Therapy Response}

In Table 1, it can be derived that the incompletely responding patients had a higher TNM status and considerably more radioiodineavid metastases than the completely responding patients. The median patient-based thyroid remnant lesion $\mathrm{AD}$ was higher for the completely responding patients $(370 \mathrm{~Gy}$ ) than the incompletely responding patients $(180 \mathrm{~Gy})$, but this was not statistically significant. For metastases, the median patient-based lesion $\mathrm{AD}$ was higher for the incompletely responding patients (63 Gy)

FIGURE 1. Box plots of lesion $A D$ for both remnants $(A-C)$ and metastases (D-F). Box plots are provided for all lesions ( $A$ and $D$ ), known-volume group ( $B$ and $E$ ), and small-volume group ( $C$ and F). Statistically significant differences are characterized by $P<0.05$. Numbers close to whiskers represent number of lesions. than the completely responding patients (39 Gy) but also not statistically significant. In 7 patients, a complete response of all radioiodine-avid lesions was seen at the lesion-based analysis. However, in the patient-based analysis these did not have a complete response because during follow-up new lesions in other anatomic areas were detected with ${ }^{18} \mathrm{~F}-\mathrm{FDG}$ PET/CT, ${ }^{124} \mathrm{I}$ PET/CT, or ultrasound in combination with cytology.
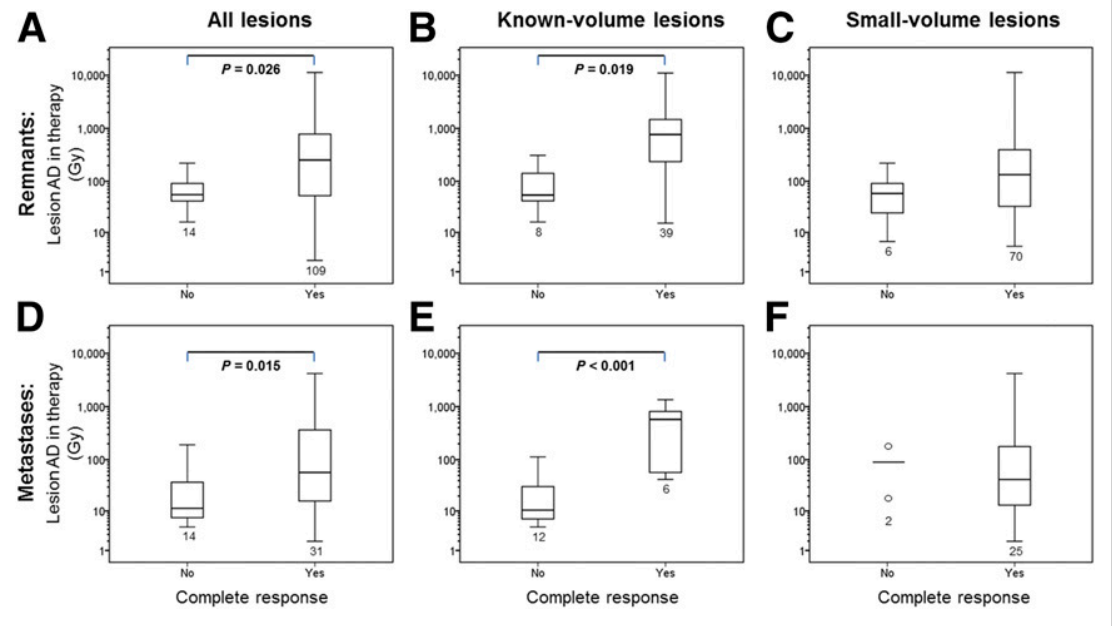

\section{DISCUSSION}

Maxon et al. $(3,4)$ demonstrated that a lesion AD threshold of 300 Gy for thyroid remnants and 85 Gy for lymph node metastases was associated with a high complete lesion response rate of $80 \%-90 \%$, establishing the lesion $\mathrm{AD}$ as an important quantity for prediction of lesion response in radioiodine treatment. Several groups 


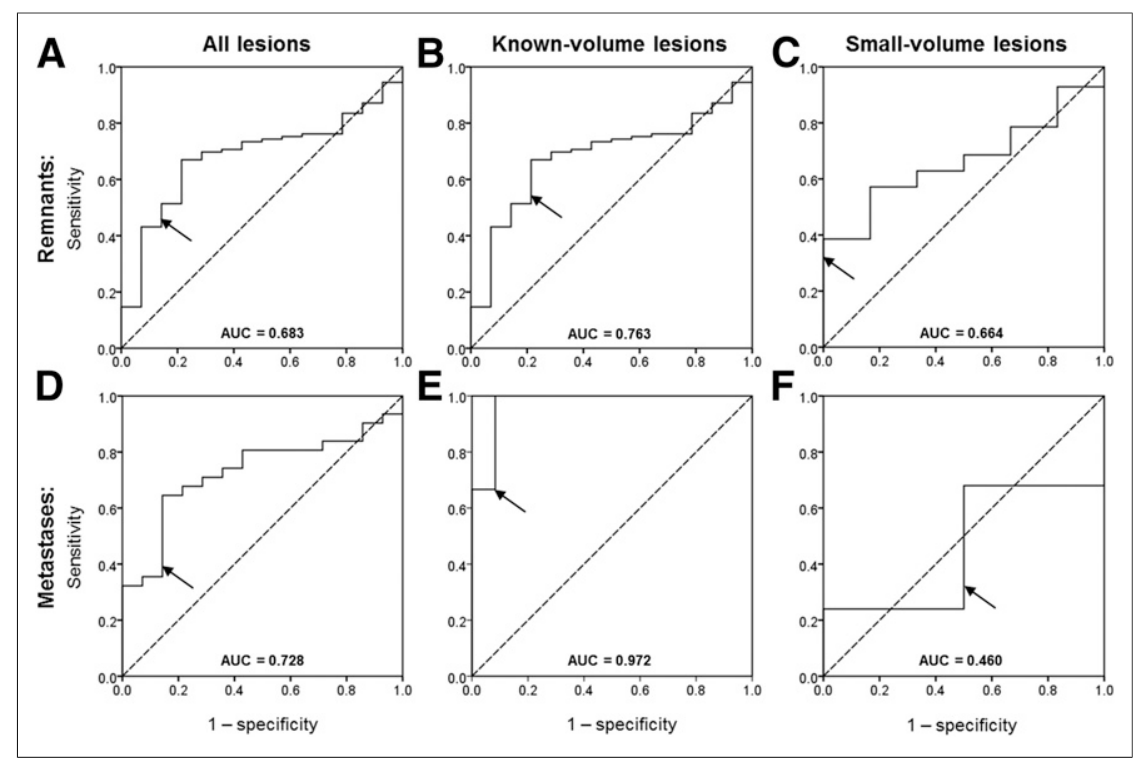

FIGURE 2. ROC curves for both remnants $(A-C)$ and metastases (D-F). ROC curves are provided for all lesions ( $A$ and $D$ ), known-volume group ( $B$ and $E$ ), and small-volume group ( $C$ and $F$ ). Arrows correspond to threshold value of $300 \mathrm{~Gy}$ for remnants and $85 \mathrm{~Gy}$ for metastases. Dashed line represents line of equality.

have identified ${ }^{124}$ I PET/CT as a promising modality for performing lesion-based dosimetry (12-17). Applying an empirically fixed radioiodine activity approach, Flower et al. (12) were the first to assess lesion AD in 3 patients using ${ }^{124}$ I PET. In agreement with our findings, they reported that the administration of fixed activities (3.0-5.5 GBq) resulted in a large variation of lesion AD to both thyroid remnants (16-400 Gy) and involved neck nodes (2.5$33 \mathrm{~Gy}$ ). In contrast, Erdi et al. (13) developed a method to estimate lesion $\mathrm{AD}$ of thyroid remnants based on pretherapeutic ${ }^{124} \mathrm{I}$ PET imaging at a maximum-tolerated activity of ${ }^{131} \mathrm{I}$ using a dose constraint of 2 Gy to blood. Performing this method in 3 patients, they observed a large lesion AD range (5-248 Gy) at maximum-tolerated activity (10-15.5 GBq). Indeed, an approach of maximum-tolerated activity applying a maximum blood dose of 2 Gy has been reported to be safe and well tolerated (25-27). Alternatively, several groups have reported an optimal activity approach using pretherapeutic ${ }^{124} \mathrm{I}$ PET/CT lesion AD calculations (14-16). In this approach, ${ }^{131}$ I therapeutic activity was chosen to achieve lesion $\mathrm{AD}$ values above the established threshold values, considering toxicity estimations. Although this approach has been reported to result in change in patient management in $25 \%-50 \%$ of DTC patients $(14,16)$, the biologic effectiveness of dosimetry-guided approaches is not proven yet (1).

To date, the only study assessing the dose-response relationship by means of ${ }^{124} \mathrm{I}$ PET/CT in a larger number of patients was recently published by Jentzen et al. (17). Therapy response for thyroid remnant and metastatic lesions above the accepted lesion AD was assessed using an optimum activity approach. This approach resulted in relatively high therapeutic ${ }^{131} \mathrm{I}$ activity (median, $10 \mathrm{GBq}$; range, 2-20 GBq), and most lesions, as expected, received an $\mathrm{AD}$ above the established threshold values. In our study, therapeutic activity was almost 4 times lower (median, $2.8 \mathrm{GBq}$; range, 1.1-5.5 GBq), and the estimated lesion $\mathrm{AD}$ range extended to the lower lesion $\mathrm{AD}$ values. As a result, we observed a statistically significant dose-response relationship, confirming a higher calculated lesion $\mathrm{AD}$ of completely responding lesions than the incompletely responding lesions. Interestingly, for thyroid rem- nants we observed a complete lesion response of $89 \%$ which is in good agreement with a complete response of $91 \%$ found by Jentzen et al. (17), whereas for metastases our study showed a complete lesion response of $69 \%$ compared with $88 \%$. These findings suggest that an optimized activity approach might be most beneficial in DTC patients presenting with metastatic disease.

Using ROC curve analysis, we showed that for lesions larger than $0.15 \mathrm{~mL}$, pretherapeutic ${ }^{124} \mathrm{I}$ PET/CT lesion dosimetry can be used as a prognostic tool to predict lesion-based ${ }^{131}$ I therapy response. For these lesions, the optimal threshold value was 90 Gy for thyroid remnants and 40 Gy for metastases. These values are considerably lower than the accepted threshold values of 300 and 85 Gy. However, it is important to note that these optimum lesion AD thresholds are defined as the values that give the maximum correct classification combining both sensitivity and specificity. In DTC patient management, a correct prediction of incompletely responding lesions is important. Consequently, for patient's management decision making, higher AD threshold values may be preferred.

Reliable lesion dosimetry requires accurate radioactivity quantification and volume estimation, which are affected by the finite spatial resolution of current nuclear medicine imaging equipment (28), in particular for the small lesion volumes often encountered in DTC patients. In addition, high-spatial-resolution images obtained by ultrasonography or CT are considered unreliable because of a lack of differentiation between thyroid tissue and hematoma on these modalities (1). As a result, lesion AD calculations are considered unreliable for small lesions. Using an established PET-based thresholding method yielding reliable volume estimation for lesions larger than $0.80 \mathrm{~mL}$, Jentzen et al. (17) were able to accurately calculate lesion $\mathrm{AD}$ for $24 \%$ and $27 \%$ of all analyzed thyroid remnants and metastases, respectively. In our study, we used a more advanced segmentation technique allowing volume estimation down to the ${ }^{124}$ I PET spatial resolution volume of $0.15 \mathrm{~mL}$. Consequently, reliable lesion $\mathrm{AD}$ estimation was obtained in $38 \%$ of thyroid remnants and $40 \%$ of metastases. Despite the improved volume segmentation technique, for most lesions only the minimum lesion $\mathrm{AD}$ could be calculated. In the future, further developments in PET/MRI might contribute to additional improvements of the dosimetry in smallvolume disease (29).

Using voxel-based 3-dimensional dose distribution calculations, several studies have reported on the AD distribution heterogeneity within lesions $(13,14)$. In these studies, substantial variability in intratumoral $\mathrm{AD}$ was observed, possibly resulting in underdosing of tumor subregions and ultimately therapy failure (13). Although in our study correction for partial-volume effect was performed by means of the recovery coefficient, heterogeneity of the lesion AD distribution was not considered because most lesions included in our study were small compared with the ${ }^{124}$ I PET spatial resolution.

A limitation of our study was its retrospective nature. However, a high concordance with other studies using ${ }^{124}$ I likely confirms the validity of our results. In fact, to date, no prospective randomized study addressing the optimal activity approach has been published, 
and the optimal therapeutic activity is not established yet $(1,2)$. The results of our study indicate that pretherapeutic ${ }^{124} \mathrm{I}$ PET/CT lesion dosimetry can be used as a prognostic tool to predict lesion response. Therefore, we would strongly advocate cooperative efforts to establish a multicenter prospective ${ }^{124}$ I trial to confirm our findings.

\section{CONCLUSION}

This study provides evidence of a statistically significant doseresponse relationship assessed by means of pretherapeutic ${ }^{124} \mathrm{I}$ PET/CT dosimetry in both thyroid remnants and metastases. This will be a clinically useful contribution in patient management.

\section{DISCLOSURE}

The costs of publication of this article were defrayed in part by the payment of page charges. Therefore, and solely to indicate this fact, this article is hereby marked "advertisement" in accordance with 18 USC section 1734. No potential conflict of interest relevant to this article was reported.

\section{REFERENCES}

1. Luster M, Clarke SE, Dietlein M, et al. Guidelines for radioiodine therapy of differentiated thyroid cancer. Eur J Nucl Med Mol Imaging. 2008;35:1941-1959.

2. Cooper DS, Doherty GM, Haugen BR, et al. Revised American Thyroid Association management guidelines for patients with thyroid nodules and differentiated thyroid cancer. Thyroid. 2009;19:1167-1214.

3. Maxon HR, Thomas SR, Hertzberg VS, et al. Relation between effective radiation dose and outcome of radioiodine therapy for thyroid cancer. N Engl J Med. 1983;309:937-941.

4. Maxon HR, Englaro EE, Thomas SR, et al. Radioiodine-131 therapy for welldifferentiated thyroid cancer: a quantitative radiation dosimetric approachoutcome and validation in 85 patients. J Nucl Med. 1992;33:1132-1136.

5. Eschmann SM, Reischl G, Bilger K, et al. Evaluation of dosimetry of radioiodine therapy in benign and malignant thyroid disorders by means of iodine-124 and PET. Eur J Nucl Med Mol Imaging. 2002;29:760-767.

6. Lassmann M, Hänscheid H, Verburg FA, Luster M. The use of dosimetry in the treatment of differentiated thyroid cancer. Q J Nucl Med Mol Imaging. 2011;55: 107-115.

7. Pentlow KS, Graham MC, Lambrecht RM, et al. Quantitative imaging of iodine124 with PET. J Nucl Med. 1996;37:1557-1562.

8. Herzog H, Tellmann L, Qaim SM, Spellerberg S, Schmid A, Coenen HH. PET quantitation and imaging of the non-pure positron-emitting iodine isotope ${ }^{124} \mathrm{I}$. Appl Radiat Isot. 2002;56:673-679.

9. Lubberink M, Herzog H. Quantitative imaging of ${ }^{124} \mathrm{I}$ and ${ }^{86} \mathrm{Y}$ with PET. Eur J Nucl Med Mol Imaging. 2011;38(suppl 1):S10-S18.

10. Jentzen W. Experimental investigation of factors affecting the absolute recovery coefficient in iodine-124 PET lesion imaging. Phys Med Biol. 2010;55: 2365-2398.
11. Preylowski V, Schlögl S, Schoenahl F, et al. Is the image quality of I-124-PET impaired by an automatic correction of prompt gammas? PLoS One. 2013;8: e71729.

12. Flower MA, Schlesinger T, Hinton PJ, et al. Radiation dose assessment in radioiodine therapy: 2-practical implementation using quantitative scanning and PET, with initial results on thyroid carcinoma. Radiother Oncol. 1989;15: 345-357.

13. Erdi YE, Macapinlac H, Larson SM, et al. Radiation dose assessment for I-131 therapy of thyroid cancer using I-124 PET imaging. Clin Positron Imaging. 1999;2:41-46.

14. Sgouros G, Kolbert KS, Sheikh A, et al. Patient-specific dosimetry for ${ }^{131} \mathrm{I}$ thyroid cancer therapy using ${ }^{124} \mathrm{I}$ PET and 3-dimensional-internal dosimetry (3D-ID) software. J Nucl Med. 2004;45:1366-1372.

15. Freudenberg LS, Jentzen W, Görges R, et al. ${ }^{124}$ I-PET dosimetry in advanced differentiated thyroid cancer: therapeutic impact. Nuklearmedizin. 2007;46: 121-128.

16. Pettinato C, Spezi E, Nanni C, et al. Pretherapeutic dosimetry in patients affected by metastatic thyroid cancer using ${ }^{124}$ I PET/CT sequential scans for ${ }^{131} \mathrm{I}$ treatment planning. Clin Nucl Med. 2014;39:e367-e374.

17. Jentzen W, Hoppenbrouwers J, van Leeuwen P, et al. Assessment of lesion response in the initial radioiodine treatment of differentiated thyroid cancer using ${ }^{124}$ I PET imaging. J Nucl Med. 2014;55:1759-1765.

18. Jentzen W. An improved iterative thresholding method to delineate PET volumes using the delineation-averaged signal instead of the enclosed maximum signal. J Nucl Med Technol. 2015;43:28-35.

19. Greene FL, Compton CC, Fritz AG, Shah JP, Winchester DP. AJCC Cancer Staging Atlas. New York, NY: Springer; 2006.

20. Jentzen W, Freudenberg L, Bockisch A. Quantitative imaging of ${ }^{124}$ I with PET/ CT in pretherapy lesion dosimetry effects impairing image quantification and their corrections. Q J Nucl Med Mol Imaging. 2011;55:21-43.

21. Jentzen W, Freudenberg L, Eising E, Sonnenschein W, Knust J, Bockisch A. Optimized ${ }^{124} \mathrm{I}$ PET dosimetry protocol for radioiodine therapy of differentiated thyroid cancer. J Nucl Med. 2008;49:1017-1023.

22. Stabin MG, Sparks RB, Crowe E. OLINDA/EXM: the second-generation personal computer software for internal dose assessment in nuclear medicine. $\mathrm{J} \mathrm{Nucl}$ Med. 2005;46:1023-1027.

23. Hanley JA, McNeil BJ. The meaning and use of the area under a receiving operating characteristic (ROC) curve. Radiology. 1982;143:29-36.

24. Youden WJ. An index for rating diagnostic tests. Cancer. 1950;3:32-35.

25. Lassmann M, Hänscheid H, Chiesa C, Hindorf C, Flux G, Luster M. EANM dosimetry committee series on standard operational procedures for pre-therapeutic dosimetry I: blood and bone marrow dosimetry in differentiated thyroid cancer therapy. Eur J Nucl Med Mol Imaging. 2008;35:1405-1412.

26. Verburg FA, Biko J, Diessl S, et al. I-131 activities as high as safely administrable (AHASA) for the treatment of children and adolescents with advanced differentiated thyroid cancer. J Clin Endocrinol Metab. 2011;96:E1268-E1271.

27. Verburg FA, Hänscheid H, Biko J, et al. Dosimetry-guided high-activity ${ }^{131} \mathrm{I}$ therapy in patients with advanced differentiated thyroid carcinoma: initial experience. Eur J Nucl Med Mol Imaging. 2010;37:896-903.

28. Wierts R, de Pont CD, Brans B, Mottaghy FM, Kemerink GJ. Dosimetry in molecular nuclear therapy. Methods. 2011;55:196-202.

29. Nagarajah J, Jentzen W, Hartung V, et al. Diagnosis and dosimetry in differentiated thyroid carcinoma using ${ }^{124}$ I PET: comparison of PET/MRI vs PET/CT of the neck. Eur J Nucl Med Mol Imaging. 2011;38:1862-1868. 